\title{
La petite irrigation par les eaux souterraines, une solution durable contre la pauvreté et les crises alimentaires au Niger ?
}

\author{
Yahaya Nazoumou $^{1, *}$, Guillaume Favreau ${ }^{2}$, Mahaman Moustapha Adamou ${ }^{3}$ et Ibrahim Maïnassara ${ }^{4}$ \\ ${ }^{1}$ Université Abdou Moumouni, Faculté des Sciences \& Techniques, Département de Géologie, Campus universitaire Rive droite, \\ 10662 Niamey, Niger \\ ${ }^{2}$ UMR 5569 HydroSciences Montpellier (HSM), Institut de Recherche pour le Développement (IRD), Montpellier, France \\ ${ }^{3}$ Université Abdou Moumouni de Niamey, Faculté d'Agronomie, Niamey, Niger \\ ${ }^{4}$ Institut de Recherche pour le Développement (IRD), Représentation au Niger, Niamey, Niger
}

\begin{abstract}
Résumé - Dans les pays du Sahel, le développement de l'agriculture irriguée est une des solutions pour améliorer la sécurité alimentaire. À travers l'exemple du sud-ouest du Niger, cette étude montre que le développement d'une irrigation à faible coût est possible par pompage de l'eau des nappes phréatiques, ressource renouvelable et mieux répartie dans l'espace que les eaux de surface. Les ressources en eau et en terres irrigables de la zone ont été localisées, quantifiées et leur potentiel à long terme réévalué à partir de données actualisées. Les résultats montrent que 50000 à 160000 ha (3 à $9 \%$ de la surface totale cultivée) pourraient être mis en valeur par la petite irrigation à partir des eaux souterraines les plus accessibles (jusqu'à $20 \mathrm{~m}$ de profondeur). Cette estimation est du même ordre de grandeur que celle déjà avancée pour les seules eaux de surface, doublant ainsi le potentiel irrigable de la zone.
\end{abstract}

Mots clés : eau souterraine / ressource en sol / irrigation / pauvreté / crise alimentaire

\begin{abstract}
Small irrigation from shallow groundwater, a sustainable solution to alleviate poverty and food crises in Niger? In Sahelian countries, the development of irrigated agriculture is one of the solutions to avoid repetitive food crises. Considering south-western Niger as a regional case study, this paper demonstrates that increasing low-cost groundwater irrigation represents a long-term solution, using shallow, unconfined perennial groundwater, widely distributed in this region. Groundwater resources and soil characteristics were described and localized in space, quantified in volume and/or surface area, and their longterm potential estimated as a function of updated datasets. Data analysis demonstrates that $\sim 50,000$ to 160,000 ha ( 3 to $9 \%$ of present-day cultivated areas) could be turned into small irrigated fields using accessible shallow groundwater (water table depth $\leq 20 \mathrm{~m}$ ). This would double the regional capacity for irrigation.
\end{abstract}

Keywords: groundwater / soil resource / irrigation / poverty / food insecurity

\section{Introduction}

La zone sahélienne est caractérisée par des précipitations faibles ( 300 à $700 \mathrm{~mm} / \mathrm{an}$ ) avec une forte variabilité interannuelle et intra-saisonnière. L'évapotranspiration potentielle très importante $(\sim 2500 \mathrm{~mm} / \mathrm{an})$ rend le déficit hydrique chronique. Les systèmes agraires y sont basés sur l'agriculture pluviale de subsistance et l'élevage extensif, qui sont très vulnérables aux aléas climatiques. En années de sécheresse, le déficit de production agricole entraîne des crises alimentaires, avec des famines qui peuvent être dramatiques (1913, 1973 et 1984).

Pays sahélien enclavé, le Niger illustre bien la problématique de sécurité alimentaire : une population avec un taux d'accroissement de $3,9 \%$ en 2012 , vivant à $80 \%$ de l'agriculture et de l'élevage, une pression forte sur des terres

$\overline{\text { *Auteur de correspondance }}$ : nazoumou@gmail.com agricoles vulnérables à l'érosion qui conduit à un appauvrissement des sols, une irrégularité des rendements et de la production agricole qui entraîne des déficits céréaliers parfois importants (FAO, 2003 ; MAG, 2015 ; Stoorvogel et Smaling, 1990). Depuis les grandes crises sahéliennes des années 1970 et 1980, le Niger fait régulièrement face à des crises alimentaires nationales : 1991, 2005 et 2010 (Bonnecase, 2010 ; Gado, 1993 ; Michiels et al., 2012).

L'autosuffisance alimentaire est donc au cœur des stratégies de développement du pays. L'irrigation est considérée comme le meilleur moyen d'augmenter la production agricole et de réduire sa vulnérabilité face à la variabilité climatique. Avec l'appui des bailleurs de fonds internationaux, l'État a lourdement investi dans la grande irrigation, dont les superficies sont passées de 350 ha en 1934 à 13000 ha en 2012 (CEIPI, 2011 ; MAG, 2015). Quatre-vingts pour cent ont été aménagées entre 1970 et 1990. Les périmètres irrigués sont 


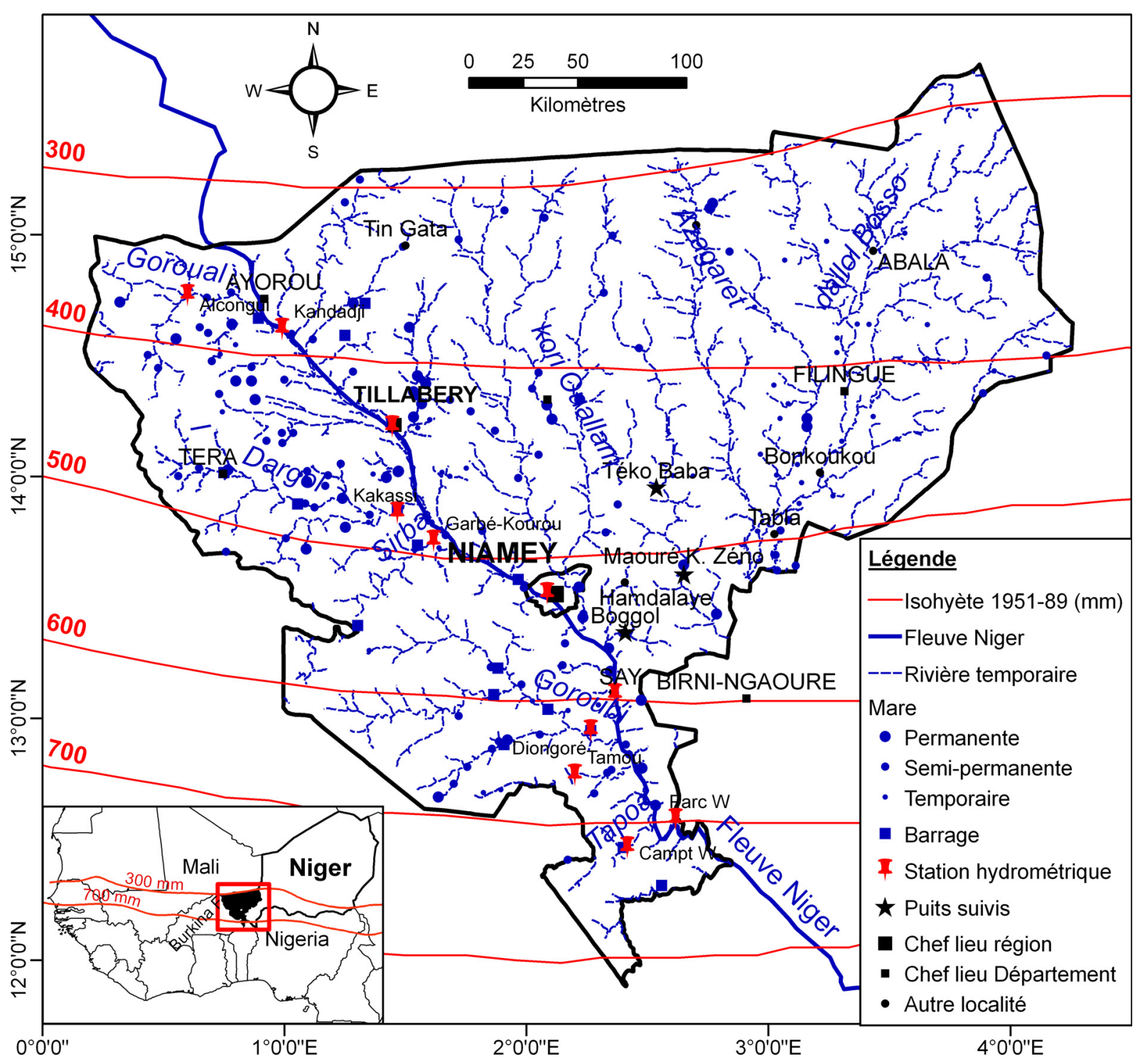

Fig. 1. Réseau hydrographique et isohyètes 1951-1989 du SO du Niger, région de Tillabéry.

Fig. 1. Hydrographic network and isohyets 1951-1989 of the SW of Niger.

concentrés dans les vallées où les potentialités en eau de surface sont importantes. La riziculture est largement dominante. La production a atteint un record de 80000 tonnes en 1996. Mais la contribution de la riziculture à la production céréalière reste faible ( $2 \%)$. En outre, le riz est surtout consommé en ville, et ne couvre que $3 \%$ des besoins nationaux en céréales (BM, 2009). À partir de 1984, des périmètres irrigués collectifs dits de contre-saison ont été encouragés pour assurer une production locale et accroître les revenus en milieu rural. Ces aménagements se sont surtout développés à partir des eaux souterraines peu profondes, dans les vallées et les bas-fonds (ARID, 2004).

La libéralisation économique des années 1990 a entraîné d'importants changements dans le secteur irrigué au Sahel (Barbier et al., 2011) : le développement de la grande irrigation a été ralenti, la gestion des périmètres publics transférée aux exploitants, les filières de commercialisation privatisées (Cochand, 2007), favorisant l'éclosion d'une petite irrigation privée informelle mais plus dynamique.
Le potentiel irrigable du Niger, évalué à 270000 ha à partir des seules eaux de surface (SOGREAH/BRGM, 1981), n'a jamais été réévalué depuis trois décennies (FAO, 1997 ; MHE/ LCD, 1999 ; FAO, 2002, 2015). Seulement un tiers de ce potentiel est exploité (FAO, 2005). Pour développer l'irrigation, les eaux souterraines, mieux réparties dans l'espace, pourraient également être mobilisées, là où des terres irrigables sont aussi disponibles. C'est cette opportunité qui est discutée ici à travers l'exemple de la région de Tillabéry.

\section{Matériel et méthodes}

La région de Tillabéry occupe la majeure partie du sudouest du pays (Fig. 1) et s'étend de part et d'autre du fleuve Niger. La rive droite (RD), constituée de massifs géologiques cristallins (Machens, 1973), est exclue de l'étude car elle ne recèle que peu de ressources en eau souterraine et que les données y sont rares. En revanche, la rive gauche (RG) est 
sédimentaire et abrite la nappe phréatique continue du Continental Terminal 3 (CT3) pour laquelle des données sont disponibles ; elle constituera notre zone d'étude.

Cette étude se base sur la documentation et les données collectées et archivées par différents acteurs du secteur de l'irrigation au Niger (Favreau et Nazoumou, 2010). Les études hydrogéologiques majeures sont celles issues des travaux de Boeckh (1965) et de Greigert et Bernert (1979). Elles ont notamment fourni de précieux historiques de niveau des nappes entre 1950 et 1979, qui complètent les mesures effectuées entre 1990 et 2012 (Favreau et al., 2012 ; Leduc et al., 2001). Ces dernières sont des informations fiables, traitées et analysées régulièrement. Les données de près de 4500 points d'eau (puits, forages, mares) ont été extraites de la base de données d'inventaire des ressources hydrauliques du ministère de l'Hydraulique du Niger (MHE/LCD). Leur qualité étant parfois incertaine et les mises à jour peu régulières, un dépouillement et un contrôle des données ont été nécessaires. Les chroniques piézométriques ont permis une analyse à long terme de l'évolution des ressources en eau dans la nappe phréatique du CT3. La carte piézométrique de Greigert et Bernert (1979) a été numérisée et les valeurs interpolées suivant une grille de $90 \times 90 \mathrm{~m}$ à l'aide du logiciel ArcGis 9.x (ESRI $\left.{ }^{B}\right)$. Les débits spécifiques des puits et forages sont pour la plupart issus d'essais de pompage de courte durée. Leur représentativité est donc relative, mais ils permettent néanmoins d'apprécier la productivité des aquifères. Les données météorologiques (Fig. 2A) de la Direction de la Météorologie nationale du Niger ont été critiquées et traitées. Celles relatives aux débits des cours d'eau sont issues des bases de données hydrologiques du MHE/LCD et de l'Autorité du Bassin du Niger (ABN).

Les travaux de Gavaud et Boulet (1967) sont la seule référence sur les sols de la zone, avec une carte pédologique au $1 / 500000^{\mathrm{e}}$ et une description détaillée de l'aptitude des sols à l'irrigation, c'est-à-dire à porter des cultures irriguées (CILSS, 2001). Elle est estimée à travers les caractéristiques morphologiques (couleur, profondeur, texture) et physico-chimiques (réserve utile, $\mathrm{pH}$, matière organique, N-P-K, capacité d'échange cationique, somme des bases échangeables et conductivité électrique) du sol. La pente, la capacité de drainage, les teneurs en carbonate de calcium et en gypse, ainsi que l'alcalinité constituent d'autres facteurs déterminants (FAO, 1983, 1995). Trois classes ont été retenues :

- sols irrigables (très propices à modérément) ;

- sols difficilement irrigables (faiblement propices) ;

- sols non irrigables (très peu à pas propices).

La carte pédologique a été numérisée pour permettre son traitement avec ArcGis et les sols aptes à l'irrigation (irrigables à modérément irrigables) ont été identifiés, cartographiés et leur superficie estimée.

Le modèle numérique de terrain (MNT) SRTM90 (Farr et Kobrick, 2000 ; Shuttle Radar Topography Mission [SRTM]) a été utilisé pour représenter la topographie de la zone d'étude. La faible résolution spatiale de ce MNT $(90 \mathrm{~m})$ n'est pas un obstacle majeur car la zone concernée est peu accidentée (pentes $<3 \%$ ). La carte de profondeur de la nappe phréatique est élaborée maille par maille à partir du MNT et de la carte piézométrique. Le croisement des cartes d'accessibilité à l'eau souterraine et d'aptitude des sols permet enfin de calculer les superficies potentiellement irrigables selon trois classes de profondeur de la nappe : 0 à $10 \mathrm{~m}, 10$ à $20 \mathrm{~m}$ et au-delà de $20 \mathrm{~m}$. Elles tiennent compte des moyens et coûts d'exhaure dans la petite irrigation privée (Daddy Gaoh et Dassargues, 1995). Des missions de terrain ont été effectuées en septembre 2009 et d'avril à juin 2010 pour apprécier l'état de la nappe et des sols, ainsi que le développement de l'irrigation dans la zone.

\section{Principaux traits régionaux}

\subsection{Un climat semi-aride à forte variabilité}

Le climat est semi-aride, caractérisé par une courte saison des pluies (juin à septembre), suivie d'une longue saison sèche (octobre à mai). Les précipitations varient de $300 \mathrm{~mm}$ par an au nord à $800 \mathrm{~mm}$ par an au sud (Fig. 1). Environ $90 \%$ du cumul annuel est relevé entre juillet et septembre. La forte disparité interannuelle se traduit par un nombre de jours de pluie compris entre 29 et 63 (Le Barbé et Lebel, 1997). Elle détermine largement la quantité et la qualité de la production céréalière pluviale. Les précipitations moyennes sont plus faibles depuis 1980 .

\subsection{Une hydrologie marquée par l'endoréisme}

Le fleuve Niger est le seul cours d'eau permanent (Fig. 1). Ses affluents principaux (Gorouol, Dargol, Sirba, Goroubi, Diamangou et Tapoa) sont situés en RD. En RG, les affluents (dallol Bosso, kori de Ouallam) constituent des vallées fossiles sans écoulements continus vers le fleuve, sauf en aval où ils drainent la nappe phréatique. En amont, les eaux de ruissellement convergent vers des ravines puis se déversent dans des mares endoréiques sous lesquelles la nappe phréatique est à faible profondeur. Le déboisement accéléré depuis les années 1970 a modifié les états de surface des sols et accru le ruissellement. Il en résulte une augmentation de la densité de drainage et des débits, accompagnée d'une érosion hydrique plus intense (Bouzou Moussa et al., 2009).

\subsection{Des aquifères aux ressources abondantes}

Les vallées du dallol Bosso et des koris sont comblées par des alluvions quaternaires qui surmontent des dépôts tertiaires du Continental Terminal (CT) : (i) le CT3 (grès sablo-silteux à argileux), terme supérieur, hydrauliquement associé aux alluvions avec lesquels il renferme la nappe phréatique du CT3, et (ii) les CT1 \& 2 (alternances sablo-argileuses), qui sont des aquifères à nappes captives localement artésiennes (Greigert, 1966 ; Greigert et Bernert, 1979). Du fait de leur plus grande profondeur, les nappes captives sont exclues du champ de cette étude. La nappe phréatique est la ressource en eau la plus importante de la zone de par son volume, son renouvellement et son accessibilité. Ses eaux s'écoulent globalement du nord au sud pour se déverser par débordement dans le fleuve (Boeckh, 1965 ; Greigert et Bernert, 1979). Cette nappe est alimentée par drainage sous les zones de concentration du ruissellement (Fig. 1). Le taux de recharge a été estimé de l'ordre de $5 \mathrm{~mm}$ par an, à partir des données isotopiques sur la période 1950-1960 (Favreau et al., 2002). Toutefois, la dynamique récente de la nappe suggère que la recharge s'est accélérée ces dernières décennies pour avoisiner les $20 \mathrm{~mm}$ par an dans les années 1990. La hausse du niveau de la nappe (Fig. 2c) confirme cet 

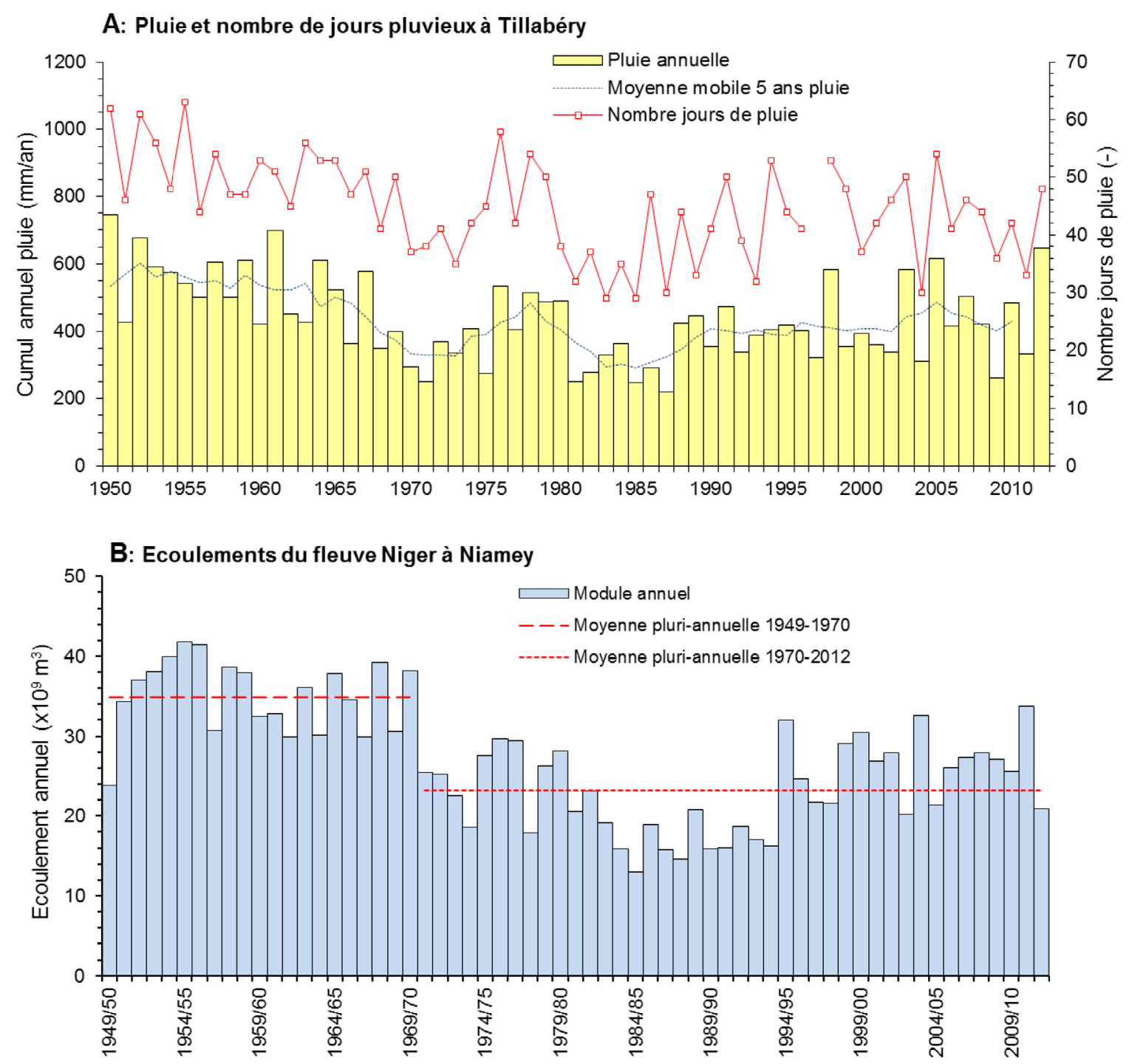

\section{C: Profondeurs de la nappe phréatique}

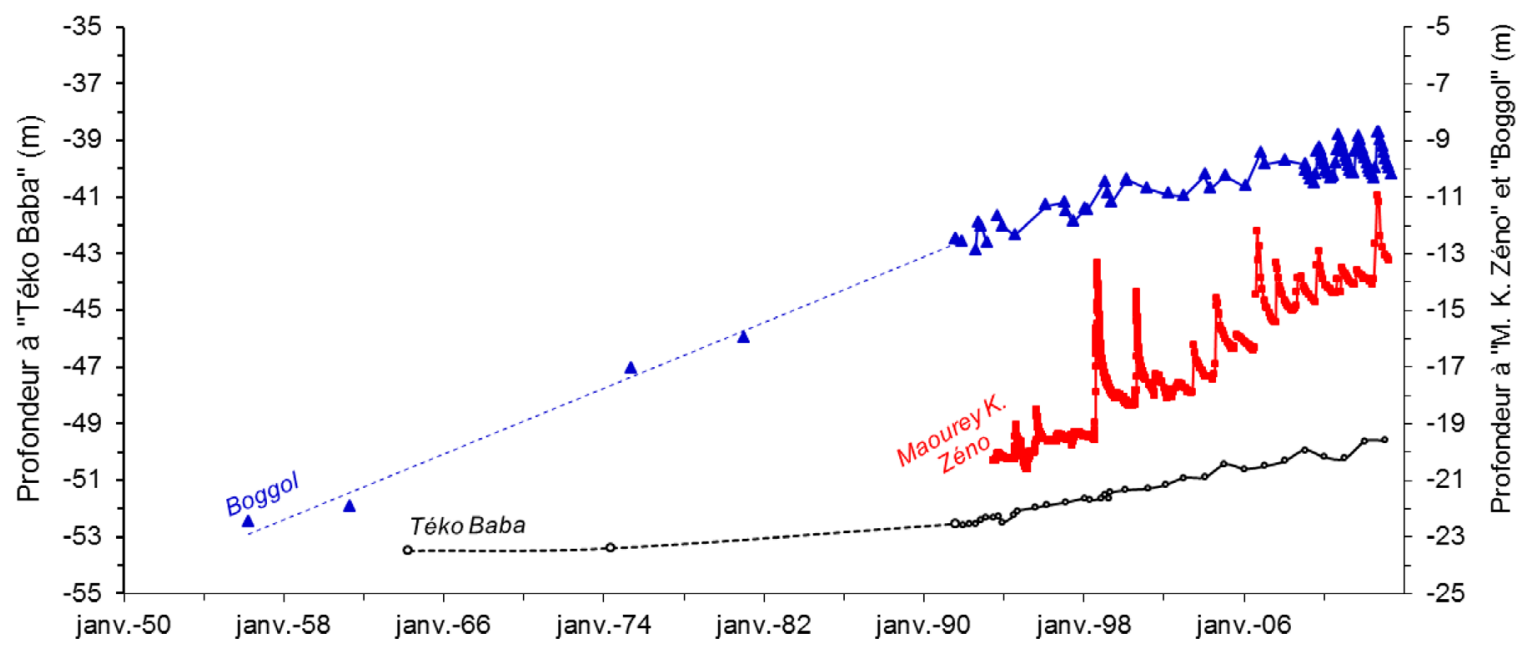

Fig. 2. Évolution pluri-décennale des ressources eau : (A) pluie à Tillabéry, (B) apports du fleuve à Niamey et (C) profondeur de la nappe phréatique du CT3 pour trois sites.

Fig. 2. Multi-decadal evolution of water resources: (A) rainfall in Tillabery, (B) Niger river discharge in Niamey and (C) depth of the CT3 water table in three places. 
Tableau 1. Description des différents types de systèmes irrigués au Niger.

Table 1. Description of several types of irrigated systems in Niger.

\begin{tabular}{ll}
\hline Type de système & Caractéristique \\
\hline $\begin{array}{l}\text { Aménagements hydro-agricoles de moyenne } \\
\text { et grande taille (AHA) }\end{array}$ & $\begin{array}{l}\text { Superficie de } 15 \text { à } 1500 \text { ha en parcelles de } 0,25 \text { à } 0,5 \text { ha. Investissement public, élevé } ; \\
\text { irrigués à partir de l'eau du fleuve (peu de nappes) par pompage, distribution gravitaire } \\
\text { de surface, riziculture et polyculture, gestion collective, encadrement de l'État }\end{array}$ \\
Petits périmètres d'irrigation privée (PIP) & $\begin{array}{l}\text { Superficie }<1 \text { ha }(0,1 \text { à } 0,75 \text { ha). Investissement privé, faible ; irrigués à partir des eaux } \\
\text { souterraines des mares ou du fleuve ; pompage motorisé, manuel ou animal ; } \\
\text { distribution manuelle, gravitaire, type californien ou goutte-à-goutte ; maraîchage }\end{array}$ \\
$\begin{array}{l}\text { Superficie de } 20 \text { à } 250 \text { ha. Investissement privé, élevé ; irrigués par pompage des eaux } \\
\text { du fleuve ou souterraines ; distribution par réseau californien et goutte-à-goutte ; } \\
\text { maraîchage et cultures de rente } \\
\text { commerciale (GMIC) }\end{array}$ & $\begin{array}{l}\text { Superficie de quelques ha. Investissement souvent public, maîtrise partielle de l'eau ; } \\
\text { irrigués à partir des eaux souterraines, rivières, mares, retenues ou en décrue } \\
\text { (zone de battement) ; gestion collective des points d'eau mais production individuelle ; } \\
\text { cultures maraîchères }\end{array}$ \\
\hline
\end{tabular}

accroissement de la recharge, avec une remontée piézométrique moyenne estimée à $4 \mathrm{~m}$ depuis 1960. Ce phénomène s'explique par le déboisement qui a provoqué une augmentation du ruissellement dans les bassins endoréiques et un afflux d'eau supplémentaire vers les zones de recharge (Favreau, 2000 ; Favreau et al., 2009).

\subsection{Des sols au potentiel irrigable contrasté}

Suivant la pluviométrie annuelle, on observe un gradient allant des sols minéraux bruts à peu évolués (isohyètes $<500 \mathrm{~mm}$ ) aux sols ferrugineux tropicaux lessivés (500 à $800 \mathrm{~mm}$ ). Entre ces deux pôles, on distingue des sols subarides tropicaux (sol brun sur matériaux argilo-sableux et sol brun-rouge sur sables éoliens), des vertisols topomorphes, des sols hydromorphes à gley ou associés à des sols ferrugineux.

Les sols minéraux bruts et les sols peu évolués n'ont pas de valeur agronomique à cause notamment de leurs faibles fertilité et capacité de rétention d'eau. Les sols subarides tropicaux, utilisés en culture pluviale, sont difficilement irrigables. Les sols brun-foncé sur formations sableuses de vallées sèches ou sur les argiles de décantation, et les sols brun-rouge minéraux évolués sur formations sableuses sont modérément propices à l'irrigation. Les vertisols, très argileux, ont une bonne fertilité et sont déjà exploités pour la riziculture. Les sols ferrugineux tropicaux sont difficilement irrigables lorsqu'ils sont peu lessivés et peu différenciés (terrasses du fleuve, vallées sèches) ou lessivés sur des formations sableuses à argilo-sableuses. Ils constituent l'essentiel des terres de culture pluviale. Les sols hydromorphes, notamment ceux sur les alluvions des affluents et ceux sur les sables d'axes de vallées fossiles, sont des terres aménageables. Ils sont exploités notamment sur les terrasses du fleuve.

\subsection{Une irrigation ancienne qui se développe}

L'irrigation est ancienne dans la vallée du fleuve Niger : le premier périmètre identifié (Koutoukalé) a été créé en 1934 (CEIPI, 2011). Mais elle a surtout été développée après les sécheresses de 1973 et 1983 . Les superficies mises en valeur en
2012 sont estimées à 107000 ha (MAG, 2015). La Stratégie de petite irrigation du Niger (SPIN) définit quatre types de systèmes irrigués (Tab. 1), en fonction des critères de maîtrise de l'eau, d'investissement, de gestion et de taille (MAG, 2015) :

- les aménagements hydro-agricoles de moyenne et grande taille (AHA) ;

- les petits périmètres d'irrigation privée (PIP) ;

- la grande et moyenne irrigation commerciale (GMIC) ;

- les périmètres de contre-saison (PCS).

Les AHA sont des périmètres irrigués à maîtrise totale de l'eau, financés et détenus par l'État et destinés à la riziculture et à la polyculture. Ils représentent $12 \%$ des superficies irriguées. Les PIP sont des périmètres individuels privés à maîtrise totale de l'eau. Ils représentent $87 \%$ des surfaces irriguées et fournissent l'essentiel de la production maraîchère. Les PCS sont des périmètres saisonniers à maîtrise partielle de l'eau, irrigués à partir des eaux souterraines (par puits ou forage) ou superficielles (rivières, mares, petites retenues), ou cultivés en décrue. Ils sont généralement aménagés par l'État pour les populations les plus vulnérables. La gestion des points d'eau est souvent commune mais les activités de production sont individuelles.

\section{Résultats}

\subsection{Une eau souterraine peu profonde, de bonne qualité et en hausse}

La profondeur de la nappe phréatique diminue du nord au sud, et des plateaux vers les vallées en fonction de la topographie (Tab. 2). Dans la vallée du dallol Bosso, cette profondeur varie de 8 à $20 \mathrm{~m}$ au nord, tandis que vers le sud elle diminue jusqu'à l'affleurement local de la nappe. Hors de la vallée, la nappe est accessible entre 20 et $90 \mathrm{~m}$ de profondeur. Les débits spécifiques des ouvrages peuvent atteindre $15 \mathrm{~m}^{3} / \mathrm{h} /$ $\mathrm{m}$ dans la partie centrale du dallol, tandis qu'au-delà, ils ne dépassent que rarement $3 \mathrm{~m}^{3} / \mathrm{h} / \mathrm{m}$. En considérant un taux de recharge variant entre 5 et $25 \mathrm{~mm}$ par an (Favreau et al., 2002), 
Tableau 2. Caractéristiques des ressources en eau souterraine de la nappe phréatique.

Table 2. Characteristics of groundwater resources of the phreatic aquifer.

\begin{tabular}{|c|c|c|c|c|}
\hline Zone & Profondeur (m) & Débit spécifique $\left(\mathrm{m}^{3} / \mathrm{h} / \mathrm{m}\right)$ & Salinité (mg/L) & Recharge (mm/an) \\
\hline Vallées dallol \& koris $^{\mathrm{a}}$ & $0-20$ & $1-15$ & $<300$ & 25 \\
\hline Autres $^{\mathrm{b}}$ & $20-90$ & $1-20$ & $30-300$ & 25 \\
\hline
\end{tabular}

${ }^{\text {a }}$ Source données : Daddy Gaoh et Dassargues (1995) et Favreau (2000).

${ }^{\mathrm{b}}$ Source données : Abdou Babaye (2013) et Favreau et al. (2009).

les réserves renouvelables de la nappe dont la superficie est d'environ $103000 \mathrm{~km}^{2}$, sont comprises entre 515 et $2575 \mathrm{hm}^{3}$ par an.

Les eaux sont douces : salinité moyenne de $\sim 100 \mathrm{mg} / \mathrm{L}$ et conductivité électrique de $\sim 250 \mu \mathrm{S} / \mathrm{cm}$ (Greigert et Bernert, 1979). Quelques valeurs supérieures à $500 \mathrm{mg} / \mathrm{L}$, souvent liées à de fortes teneurs en nitrates, sont notées au nord de Filingué et en aval dans le dallol Bosso où la nappe affleure (Daddy Gaoh et Dassargues, 1995). Le faciès dominant est bicarbonaté calcique dans la partie nord, tandis qu'au sud, les faciès sulfatés et surtout chlorurés sodiques deviennent de plus en plus dominants vers le fleuve (Favreau, 2000). Le rapport d'absorption du sodium (SAR) des eaux est de l'ordre de 3, ce qui les classe dans la catégorie des eaux à faible risque alcalin (Daddy Gaoh et Dassargues, 1995). Leur qualité n'est donc pas un facteur limitant pour l'irrigation.

\subsection{Un fort potentiel en surfaces irrigables}

La superficie des sols d'aptitude à l'irrigation très élevée à moyennement élevée est estimée à 1,13 Mha, soit $21 \%$ des $53000 \mathrm{~km}^{2}$ de la zone étudiée. Toutefois, en tenant compte de la profondeur de la nappe phréatique, le potentiel des sols facilement irrigables (nappe à moins de $10 \mathrm{~m}$ ) est de $50000 \mathrm{ha}$, soit $\sim 1 \%$ de la zone. Ces terres très favorables pour l'irrigation sont situées dans la vallée du dallol au sud de Bonkoukou (Fig. 3). Le potentiel peut être porté à environ 160000 ha (3 \%) en intégrant les zones où la nappe est entre $10 \mathrm{~m}$ et $20 \mathrm{~m}$ (nord dallol Bosso et kori de Ouallam). L'essentiel des sols classés aptes à l'irrigation sont situés dans des zones où la profondeur de l'eau est supérieure à $20 \mathrm{~m}$.

\section{Discussion}

\subsection{Variabilité et accessibilité de la ressource en eau pour l'irrigation}

La nappe libre du CT3 n'est pas la seule ressource en eau de la zone. Elle est en revanche la mieux documentée. En effet, des études menées sur cet aquifère ont permis d'accumuler des données exceptionnelles en qualité et en quantité (Favreau, 2000 ; Leduc et al., 1997). Elles ont permis de compléter les informations plus anciennes. À l'exception des aquifères alluviaux, toutes les autres nappes sont relativement profondes (>20 m). De ce fait, la nappe du CT3 est la plus importante en termes d'eau souterraine peu profonde, aisément mobilisable pour l'irrigation.

La nappe phréatique est facilement accessible dans le dallol Bosso, surtout au sud (aval) où elle se situe à moins de $10 \mathrm{~m}$.
Cela avait été consigné dès les années 1960 (Boeckh, 1965), avec une nappe qui était déjà sub-affleurante. La remontée du niveau piézométrique mise en évidence par des études récentes (Favreau et al., 2012 ; Leduc et al., 2001) est un phénomène général. Les prélèvements étant encore négligeables, de l'ordre de $0,3 \mathrm{~mm}$ par an d'après la FAO (2015), la zone de nappe à faible profondeur $(<20 \mathrm{~m})$ s'est étendue. Les remontées mesurées (Fig. 2c) à Maouré Kouara Zéno $(\sim 8 \mathrm{~m})$ et Bogol $(\sim 13 \mathrm{~m})$, deux puits situés dans les koris, témoignent de cette dynamique. L'apparition récente de nombreuses mares d'émergence de la nappe dans la partie aval du dallol (Favreau et al., 2012) constitue aussi un indicateur d'une remontée de la nappe.

Les autres aquifères alluviaux sont localisés dans les vallées des affluents RD du fleuve Niger (Gorouol, Dargol, Sirba), avec toutefois des extensions latérales très limitées. Leurs eaux sont captées entre 5 et $15 \mathrm{~m}$ de profondeur, mais les débits sont faibles (en moyenne $4 \mathrm{~m}^{3} / \mathrm{h} / \mathrm{m}$ ), et environ $18 \%$ des forages donnent des débits inférieurs à $0,5 \mathrm{~m}^{3} / \mathrm{h} / \mathrm{m}$. La recharge varie selon la pluviométrie et les écoulements des cours d'eau associés (Abdou Babaye, 2013). Ces nappes, déjà exploitées pour l'irrigation de petits périmètres, constituent des ressources additionnelles pour l'accroissement des surfaces cultivées, puisque des terres irrigables existent dans ces vallées (Gavaud et Boulet, 1967). Cependant, le fort taux d'échec des forages sur cette rive du fleuve, $\sim 40 \%$ d'après Abdou Babaye (2013), indique que les ressources en eau souterraine sont limitées par rapport à celles de la nappe phréatique du CT3 .

Le Niger constitue aussi une ressource importante pour l'irrigation. Les apports moyens annuels à Niamey (Fig. 2b) sont évalués à environ $24 \mathrm{~km}^{3}$ sur la période 1969-2012, soit une baisse de $30 \%$ par rapport à la période 1944-1969, liée à la diminution des précipitations sur le bassin supérieur (Amani et Nguétora, 2002). À partir de la date programmée de 2017, les $1,6 \mathrm{~km}^{3}$ par an du barrage de Kandadji sur le fleuve s'ajouteront aux $0,3 \mathrm{~km}^{3}$ par an mobilisés par les deux barrages de Téra et Theim sur ses affluents. Les 12 mini-barrages inventoriés (Fig. 1) totalisent $0,001 \mathrm{~km}^{3}$ par an, mais leur faible durée de vie programmée (10-15 ans), du fait de leur ensablement rapide, limite leur contribution. Les 116 mares recensées offrent aussi des eaux de qualité pendant toute ou partie de la saison sèche, mais elles sont encore mal connues en nombre et en volume (Massuel et al., 2011).

\subsection{Un potentiel irrigable sous-estimé ?}

L'évaluation complète du potentiel irrigable doit se baser sur l'adéquation entre les ressources en eau et en terres. Au Niger, les données hydrologiques et pédologiques sont souvent 


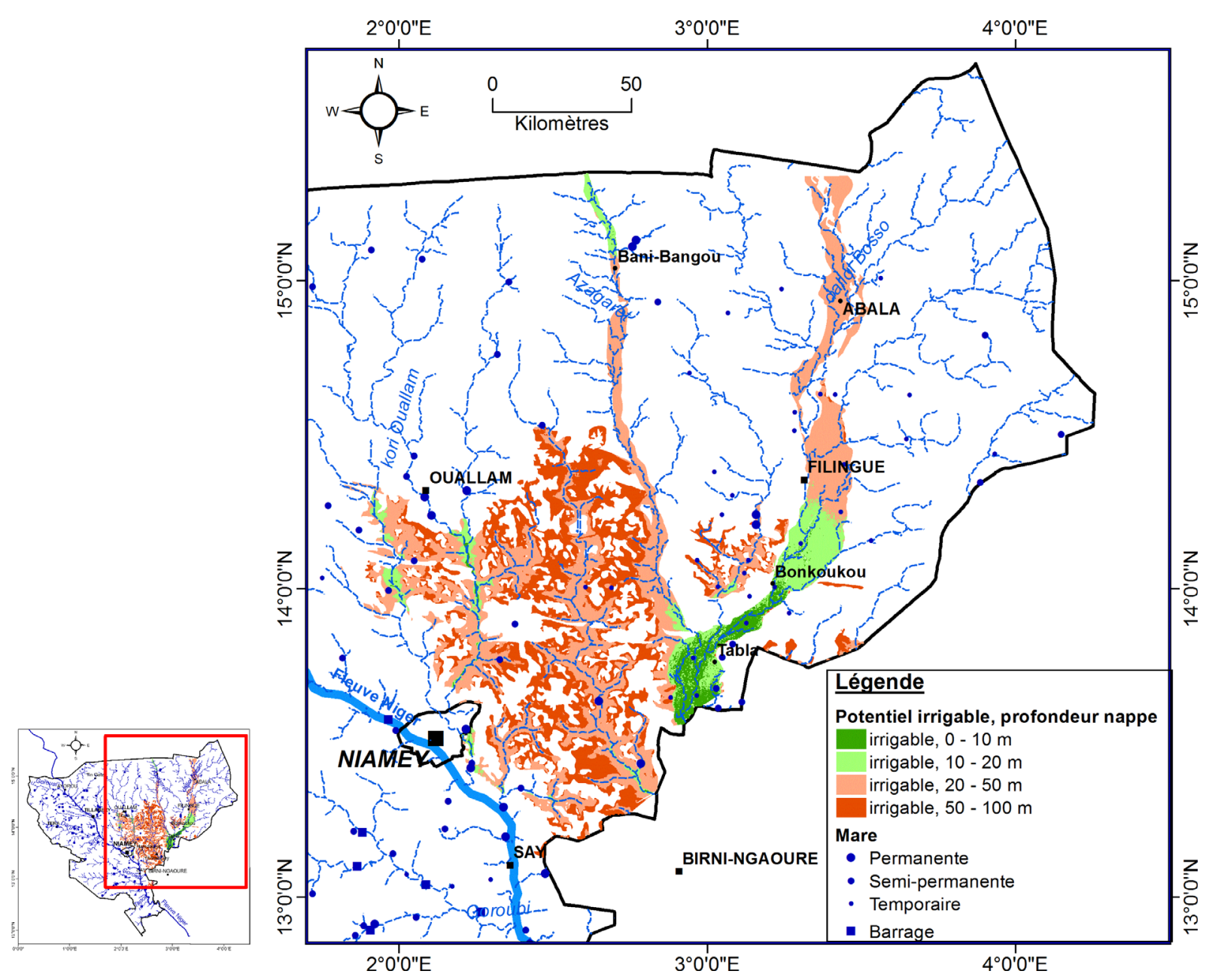

Fig. 3. Potentiel de terres irrigables suivant la profondeur de la nappe phréatique.

Fig. 3. Potential irrigable lands as function of the water table depth.

trop parcellaires pour permettre une estimation à l'échelle nationale. La plupart des études se réfèrent à des échelles souscontinentales (Rockström et al., 2007) ou à de grands bassins versants (FAO, 1997). Beaucoup de travaux ne prennent en compte qu'une partie des ressources, l'eau ou les terres (Pavelic et al., 2012) : pour évaluer le potentiel irrigable en Afrique, les rapports de la FAO $(1995,1997,2015)$ se sont basés quasiexclusivement sur les eaux de surface. La contribution des eaux souterraines n'est considérée que dans les zones semi-arides, où elle serait souvent négligeable du fait des faibles potentialités en terres irrigables.

$\mathrm{Au}$ Niger, les estimations du potentiel en terres irrigables varient entre 220000 et 440000 ha (FAO, 1997, 2002, 2015 ; MHE/LCD, 1999 ; SOGREAH/BRGM, 1981). Le chiffre officiel, repris dans les stratégies sectorielles (MAG, 2005, 2015) et par la FAO, est celui de 270000 ha, déjà avancé par SOGREAH/BRGM (1981). Mais cette estimation ancienne est discutable car réalisée sur la base des écoulements des principaux cours d'eau évalués en 1980 et d'un besoin en eau des cultures normatif de $10000 \mathrm{~m}^{3} / \mathrm{ha}$ par an $(1 \mathrm{~m} / \mathrm{an})$. L'aptitude des terres à l'irrigation, les nombreux bassins endoréiques et les ressources en eau souterraine dont les réserves renouvelables sont évaluées à 2,5 milliards de mètre cube (MHE/LCD, 1999), n'ont pas été pris en compte. On peut donc penser que le potentiel irrigable est sous-estimé, malgré, à l'inverse, la non-prise en compte du débit écologique réservé sur le fleuve et l'utilisation d'une période de référence plus humide.

Même si l'approche sols + eau utilisée ici n'est appliquée que sur une zone limitée, elle présente l'avantage d'être plus intégrée que les approches habituelles. Alors que les estimations officielles recensent seulement 10000 ha $(\sim 4 \%)$ de terres irrigables dans la vallée du dallol Bosso, nos résultats indiquent que la mobilisation des eaux souterraines peu profondes (jusqu'à $20 \mathrm{~m}$ ) permettrait d'irriguer 50000 à 160000 ha dans la zone. Toutefois, la qualité des évaluations est limitée par la précision des données sur les sols, issues d'une carte pédologique ancienne et au $1 / 500000^{\mathrm{e}}$, ce qui n'autorise qu'une classification approximative (CILSS, 2001). L'aptitude des sols a été définie à partir des besoins des grandes cultures (mil, sorgho, niébé, arachide, riz) et ne tient pas compte des besoins spécifiques des cultures maraîchères (oignon, tomate...). De plus, en 50 ans, la disparition du couvert végétal naturel et l'érosion hydrique qui affectent la zone (Leblanc et al., 2008) ont pu modifier les aptitudes des sols. En l'absence d'étude spécifique, ces conséquences sont cependant présumées réduites, car les pentes sont faibles à modérées $(0,5$ à $2,5 \%$ ) selon la classification de la FAO (1997). 

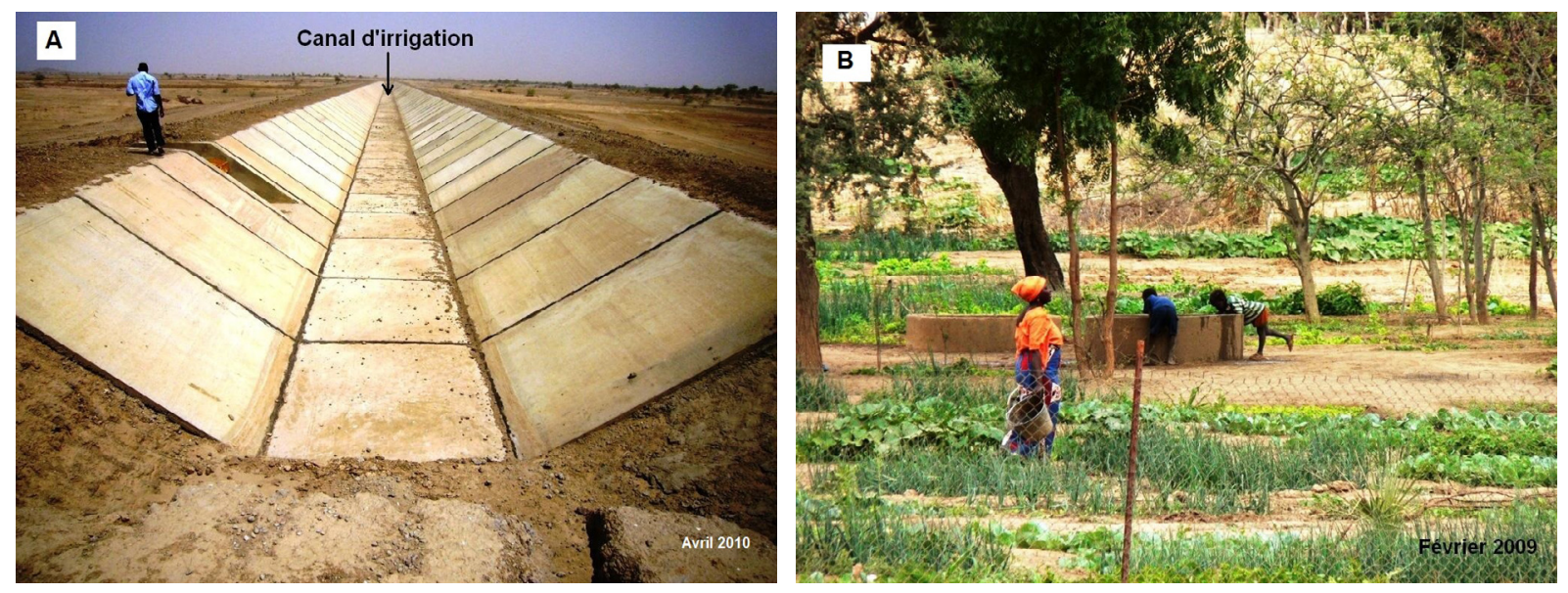

Fig. 4. Quelques périmètres irrigués : (A) à partir des eaux de surface, futur périmètre du barrage de Kandadji, (B) à partir de la nappe phréatique à Yérima Dey environ $10 \mathrm{~km}$ au SE de Maourey K. Zéno.

Fig. 4. Some irrigated schemes: (A) from surface water, the future Kandadji dam, (B) from the CT3 water table in Yérima Dey about $10 \mathrm{~km}$ SE of Maourey K. Zeno.

Tableau 3. Coûts moyens d'investissement par type de système d'irrigation au Niger.

Table 3. Average investment costs by types of irrigated systems in Niger.

Systèmes d'irrigation

Coût $\times 1000 \mathrm{FCFA} / \mathrm{ha}$

Aménagements hydro-agricoles de moyenne et grande taille

$7500-10000$

Petite irrigation privée :

a. coût faible : forage, pompe manuelle, canal terre, haie vive, clôture barbelée

b. coût moyen : forage, exhaure animale (camelin), canal revêtu plastique, clôture barbelée

c. coût élevé : puits maraîcher bétonné, groupe de pompage mobile,

$3000-3500$

$6000-6750$

réseau californien, clôture barbelée, haie vive

d. coût supérieur : puits maraîcher bétonné, groupe de pompage mobile,

$7500-9000$

goutte-à-goutte basse pression, clôture barbelée, haie vive

Grande et moyenne irrigation commerciale

Périmètres de contre-saison

Élevé et variable

$2000-5000$

Source données : BM (2009) adapté. 1000 FCFA $\approx 1,5 €$.

\subsection{Quel type d'aménagement promouvoir?}

Dans le contexte nigérien, les habitudes alimentaires des populations rurales basées sur les céréales pluviales (mil et sorgho) militent peu pour une contribution directe des cultures irriguées à la sécurité alimentaire. Toutefois, l'exportation de cultures à haute valeur ajoutée $(90 \%$ des exportations agricoles selon BM, 2009) peut garantir aux populations des revenus leur permettant d'améliorer cette sécurité. Mais l'accès aux marchés est difficile pour un pays enclavé comme le Niger, qui ne dispose que de $67 \mathrm{~km}$ de routes par $1000 \mathrm{~km}^{2}$ de terres arables (BM, 2009). Un accent particulier doit être mis sur l'amélioration des infrastructures desservant les circuits de commercialisation (MAG, 2015). L'impact sera d'autant plus important que le système d'irrigation sera petit, simple et peu coûteux. Le coût de l'eau (captage, exhaure et distribution) est en effet déterminant pour que l'irrigation soit accessible à une large population.

Les AHA (Fig. 4A) ont des coûts d'investissement (Tab. 3) hors de portée des acteurs locaux (CEIPI, 2011 ; Turral et al., 2010), et des coûts d'exploitation importants (notamment pour le pompage). Ces systèmes nécessitent en outre de grandes quantités d'eau, ce qui limite leur développement aux abords du fleuve. En dépit des réformes menées dans le secteur (Abernethy et Sally, 1999 ; Jamin et al., 2005), des difficultés persistantes de gestion (entretien, maintenance des infrastructures) limitent le développement des AHA. La monoculture, les faibles rendements, la faible taille des parcelles due à la croissance démographique, l'absence de sécurité foncière et la commercialisation des produits sont aussi des contraintes régulièrement évoquées.

La GMIC (grande irrigation privée) nécessite aussi des investissements importants et du fait de ses objectifs, son impact sur la sécurité alimentaire ou la lutte contre la pauvreté est très limité. Au contraire, l'accaparement des terres qu'elle induit (Brondeau, 2011) est une source supplémentaire d'insécurité pour les populations rurales.

Les PIP (petits privés) et les PCS (contre-saison) (Fig. 4B) connaissent un développement rapide, grâce à l'appui de l'État, sous forme de prêts ou de subventions (ARID, 2004 ; BM, 2009 ; MAG, 2015), et à leurs performances encourageantes. Les coûts d'investissement sont les plus faibles (Tab. 3), et la mise en place d'un crédit rural tend à accroître leur développement. L'environnement agro-écologique et la proximité de marchés orientent beaucoup les spéculations et les 
techniques. Pour le captage de la nappe, les forages manuels ou mécanisés avec des tubages en PVC et les puits maraîchers bétonnés sont appropriés. Quant à l'exhaure, des pompes manuelles (à pédale ou à main), dont les techniques de fabrication sont localement maîtrisées, s'adaptent bien aux petites parcelles. De plus, la baisse constatée du prix des motopompes mobiles (CEIPI, 2011) leur est aussi favorable.

\section{Conclusion}

Les crises alimentaires survenues au Niger durant les deux dernières décennies sont révélatrices des limites des formes actuelles d'irrigation pour améliorer l'autosuffisance alimentaire. Depuis 1960, comme ailleurs au Sahel, les investissements ont surtout été axés sur la grande irrigation rizicole le long des grands cours d'eau. Malgré l'importance des ressources en eau souterraine, leur valorisation par l'irrigation reste encore marginale (Diaw et al., 2003 ; FAO, 2015 ; Siebert et al., 2010). Au Niger, le volume prélevé est évalué à $20 \%$ des ressources renouvelables (MHE/LCD, 1999), alors que les terres aptes à l'irrigation sont largement disponibles et sont moins limitantes que l'accessibilité à l'eau.

L'analyse croisée des ressources en eau souterraine et en sols dans le sud-ouest du Niger indique que des potentialités durables d'irrigation existent à partir de la nappe phréatique. Son taux de recharge est élevé et ses réserves sont en hausse malgré des précipitations très fluctuantes. L'approche utilisée fournit une première évaluation du potentiel irrigable dans une des régions du Sahel les plus sensibles aux crises alimentaires et incite à une réévaluation du potentiel national d'irrigation admis depuis 1980.

L'irrigation par les eaux souterraines permet d'identifier un potentiel productif dans l'intérieur du pays par la mise en valeur de plus de terres. Toutefois, les paysans ayant des capacités d'investissement limitées, cela ne pourra être efficace en termes de sécurité alimentaire que si le système d'irrigation adopté est peu coûteux et donc accessible au plus grand nombre, et qu'il existe des marchés de proximité pour écouler les productions. Des projets pilotes de petite irrigation privée ont montré qu'en favorisant l'accès au crédit rural pour le financement de technologies à faibles coûts, il est possible d'augmenter significativement la production et d'améliorer la rentabilité des différentes cultures (CEIPI, 2011). La promotion de la petite irrigation privée à partir des eaux souterraines peu profondes est économiquement envisageable car les coûts des ouvrages de captage et des moyens d'exhaure sont réduits. Depuis 1990, les PIP suscitent un engouement certain dans le pays (Torou et al., 2013). Il s'agit de stimuler cette dynamique à travers des politiques appropriées ; l'adoption en 2015 de la SPIN s'inscrit bien dans cette logique.

Remerciements. Ce travail a été réalisé dans le cadre du programme de lutte contre la pauvreté (LUCOP) financé par la Coopération allemande (GIZ) au Niger. Les auteurs remercient la GIZ et l'Institut de recherche pour le développement (IRD) à Niamey pour avoir permis l'exécution de cette étude. Certaines données utilisées ont été acquises par la DMN et le MHE/LCD. D'autres sont issues de l'observatoire AMMA-CATCH (http://www.amma-catch.org) Niger. Cet article a été rédigé grâce au soutien de l'IRD dans le cadre de séjours d'échanges scientifiques et technologiques à HydroSciences, Montpellier (France).

\section{Références}

Abdou Babaye MS. 2013. Évaluation des ressources en eau souterraine dans le bassin de Dargol (Liptako - Niger). Thèse de doctorat, Université de Liège, Belgique.

Abernethy CL, Sally MH. 1999. Experiences of some governmentsponsored organisations of irrigators in Niger and Burkina Faso, West Africa. In: Abernethy CL, Heim F, éds. Irrigators' organisations : Government actions towards effective irrigators' organisations. Feldafing (Germany): German Foundation for International Development.

Amani A, Nguétora M. 2002. Évidence d'une modification du régime hydrologique du fleuve Niger à Niamey. In: Van Lannen H, Demuth S, eds. In Proceedings of FRIEND 2002 Conference "Regional hydrology: bridging the gap between research and practice, proceedings", Cape Town. IAHS Publ. 274:449-456.

ARID. 2004. Typologie des systèmes irrigués en Afrique de l'Ouest sahélienne. Projet Appia. Ouagadougou: ARID. http://www.aridafrique.org/IMG/pdf/Typologie_des_systemes_irrigues_AO_.pdf. Site Web accédé le 15/05/2015.

Barbier B, Ouedraogo H, Dembélé Y, Yacouba H, Barry B, Jamin JY. 2011. L'agriculture irriguée dans le Sahel ouest-africain. Cah. Agric. 20: 24-33. doi:10.1684/agr.2011.0475.

BM. 2009. Développement de l'irrigation au Niger : diagnostic et options stratégiques. Rapport 49379 NE Word Bank, Banque Mondiale, Niamey.

Boeckh E. 1965. Contribution à l'étude hydrogéologique de la zone sédentaire de la république du Niger. Dakar: Éditions BRGM.

Bonnecase V. 2010. Retour sur la famine au Sahel du début des années 1970 : la construction d'un savoir de crise. Polit. Afr. 119: 23-42.

Bouzou Moussa I, Faran Maiga O, Karimou Ambouta JM, Sarr B, Descroix L, Adamou MM. 2009. Les conséquences géomorphologiques de l'occupation des sols et des changements climatiques dans un bassin versant rural sahélien. Secheresse 20: 145-152. doi: $10.1684 / \mathrm{sec} .2009 .0163$.

Brondeau F. 2011. L'agrobusiness à l'assaut des terres irriguées de l'Office du Niger (Mali). Cah. Agric. 20: 136-143. doi :10.1684/ agr.2011.0472.

CEIPI. 2011. Projets et programmes de développement de l'irrigation au Niger (1960-2010) : éléments pour un bilan. Centre d'Études et d'Information sur la Petite Irrigation (CEIPI). Niamey : http://www. reca-niger.org/IMG/pdf/Rapport_CEIPI_Niger_1960-2010.pdf. Site Web accédé le 15/05/2015.

CILSS. 2001. Les aptitudes agricoles et pastorales des sols dans les pays du CILSS. AGRHYMET, Niamey : http://orbi.ulg.be/ bitstream/2268/72732/1/LesSolsDesPaysDuCILSS.pdf. Site Web accedé le 17/05/2015.

Cochand J. 2007. La petite irrigation privée dans le sud Niger : potentiels et contraintes d'une dynamique locale. Le cas du sud du Département de Gaya. Mémoire, Université de Lausane, Suisse.

Daddy Gaoh A, Dassargues A. 1995. Exploitation de la nappe alluviale du dallol Maouri pour des cultures irriguées au Niger. Secheresse 6: 257-263.

Diaw BEH, Ackerer P, Boivin P, Laval F. 2003. Suivi expérimental des transferts d'eau provoqués par l'irrigation dans le périmètre de Ouro Madiou en moyenne vallée du fleuve Sénégal. Cah. Agric. 12: 103-110.

FAO. 1983. Land evaluation for rainfed agriculture. Rome: FAO Soils Bulletin $\mathrm{N}^{\circ} 52$.

FAO. 1995. Irrigation in Africa in figures / L'irrigation en Afrique en chiffres. Rome: FAO editions.

FAO. 1997. Irrigation potential in Africa. A basin approach. Rome: FAO editions. 
FAO. 2002. Niger - Stratégie nationale de développement de l'irrigation et de collecte des eaux de ruissellement. Rome: FAO editions.

FAO. 2003. Gestion de la fertilité des sols pour la sécurité alimentaire en Afrique subsaharienne. Rome: FAO editions.

FAO. 2005. L'irrigation en Afrique en chiffres - Enquête AQUASTAT 2005. Rome: FAO Rapports sur l'eau $\mathrm{N}^{\circ} 29$.

FAO. 2015. AQUASTAT website, Food and Agriculture Organization of the United Nations (FAO). http://www.fao.org/nr/aquastat. Website accessed on 2015/05/15.

Farr TG, Kobrick M. 2000. Shuttle Radar Topography Mission produces a wealth of data. Am. Geophys. Union Newspaper Eos 81: 583-585.

Favreau G. 2000. Caractérisation et modélisation d'une nappe phréatique en hausse au Sahel. Thèse de doctorat, Paris XI Orsay, France.

Favreau G, Nazoumou Y. 2010. Ressources en eau dans la région de Tillabéri (Niger). Potentiel de mise en valeur par l'agriculture irriguée. Niamey: GIZ éditions.

Favreau G, Leduc C, Marlin C, et al. 2002. Estimate of recharge of a rising water table in semiarid Niger from H-3 and C-14 modeling. Ground Water 40: 144-151. doi:10.1111/j.1745-6584.2002. tb02499.x.

Favreau G, Cappelaere B, Massuel S, et al. 2009. Land clearing, climate variability and water resources increase in Southwest Niger: a review. Water Res. Res. 45: W00A16. doi:10.1029/2007WR006785.

Favreau G, Nazoumou Y, Leblanc M, Guéro A, Baba Goni I. 2012. Groundwater resources increase in the Iullemmeden Basin, West Africa. In: Treidel H, Martin-Bordes JL, Gurdak JJ, eds. Climate Change Effects on Groundwater Resources: A Global Synthesis of Findings and Recommendations. CRC Press/Balkema/ Taylor \& Francis. IAH Blue Book Series Publi. 2012;27:113128.

Gado BA. 1993. Une histoire des famines au Sahel. Étude des grandes crises alimentaires $\left(\mathrm{XIX}^{\mathrm{e}}-\mathrm{XX}^{\mathrm{e}}\right.$ siècles). Paris: L'Harmattan.

Gavaud M, Boulet R. 1967. Carte pédologique de reconnaissance de la République du Niger 1:500000, Feuille Niamey. Paris: éditions ORSTOM.

Greigert J. 1966. Description des formations crétacées et tertiaires du bassin des Iullemmeden (Afrique occidentale). Paris: éditions BRGM.

Greigert J, Bernert G. 1979. Atlas des eaux souterraines de la république du Niger. État des connaissances. 79 AGE 00 1. Orléans: éditions BRGM.

Jamin JY, Bisson P, Fusillier JL, et al. 2005. La participation des usagers à la gestion de l'irrigation : des mots d'ordre aux réalités dans les pays du Sud. Colloque Irrigation et développement durable, 2005-05-19, Paris. Les colloques de l'Académie d'agriculture de France 91: 65-83.

Le Barbé L, Lebel T. 1997. Rainfall climatology of the HAPEX-Sahel region during the years 1950-1990. J. Hydrol. 188-189: 43-73.
Leblanc M, Favreau G, Massuel S, Tweed S, Loireau M, Cappelaere B. 2008. Land clearance and hydrological change in the Sahel: SW Niger. Global Planetary Change 61: 135-150. doi:10.1016/j. gloplacha.2007.08.011.

Leduc C, Bromley J, Schroeter P. 1997. Water table fluctuation and recharge in semi-arid climate: some results of the HAPEX-Sahel hydrodynamic survey (Niger). J. Hydrol. 188-189: 123-138.

Leduc C, Favreau G, Schroeter P. 2001. Long-term rise in a Sahelian water-table: The Continental Terminal in south-west Niger. $J$. Hydrol. 243: 43-54. doi:10.1016/S0022-1694(00)00403-0.

Machens E. 1973. Contribution à l'étude des formations du socle cristallin et de la couverture sédimentaire de l'ouest de la république du Niger. Niamey: éditions BRGM.

MAG. 2005. Stratégie nationale de développement de l'irrigation et de la collecte des eaux de ruissellement (SNDI/CER, 2005). Niamey: éditions Ministère de l'Agriculture.

MAG. 2015. Stratégie de la Petite Irrigation du Niger (SPIN). Niamey: éditions Ministère de l'Agriculture.

Massuel S, Cappelaere B, Favreau G, Leduc C, Lebel T, Vischel T. 2011. Integrated surface water-groundwater modelling in the context of increasing water reserves of a regional Sahelian aquifer. Hydrol. Sci. J. 56: 1242-1264. doi:10.1080/02626667.2011. 609171.

MHE/LCD. 1999. Schéma Directeur de mise en valeur et de gestion des ressources en eau du Niger. Niamey: éditions MHE/LCD.

Michiels D, Egg J, Blein R. 2012. La répétition des crises alimentaires et nutritionnelles au Niger : la rénovation urgente des politiques de sécurité alimentaire. Cah. Agric. 21: 302-310. doi:10.1684/ agr.2012.0588.

Pavelic P, Smakhtin V, Favreau G, Villholth KG. 2012. Water balance approach for assessing potential for smallholder groundwater irrigation in Sub-Saharan Africa. Water SA 38-3: 399-406. doi:10.4314/wsa.v38i3.5.

Rockström J, Lannerstad M, Falkenmark M. 2007. Assessing the water challenge of a new green revolution in developing country. Proc. Natl Acad. Sci. USA 104: 6253-6260.

Siebert S, Burke J, Faures JM, et al. 2010. Groundwater use for irrigation - a global inventory. Hydrol. Earth Syst. Sci. 14: 1863-1880. doi:10.5194/hess-14-1863-2010.

SOGREAH/BRGM. 1981. Étude du plan de développement de l'utilisation des ressources en eau du Niger. Niamey: éditions BRGM.

Stoorvogel JJ, Smaling EMA. 1990. Assessment of soil nutrient depletion in Sub-Saharan Africa 1983-2000. Volume 1 Main report. Wageningen (The Netherlands), The Wind and Staring Centre. Report 28, 4 Volumes.

Torou BM, Favreau G, Barbier B, Pavelic P, Illou M, Sidibé F. 2013. Constraints and opportunities for groundwater irrigation arising from hydrologic shifts in the Iullemmeden basin, southwestern Niger. Water Int. 38(4): 465-479. doi:10.1080/02508060.2013.817042.

Turral H, Svendsen M, Faures JM. 2010. Investing in agriculture : reviewing the past and looking to the future. Agric. Water Manage. 97: 551-560. doi:10.1016/j.agwat.2009.07.012.

Cite this article as: Nazoumou Y, Favreau G, Adamou MM, Maïnassara I. 2016. La petite irrigation par les eaux souterraines, une solution durable contre la pauvreté et les crises alimentaires au Niger? Cah. Agric. 25: 15003. 